\title{
Effect of acupuncture on insomnia in menopausal women: a study protocol for a randomized controlled trial
}

\author{
Shanshan $\mathrm{Li}^{1+}$, Ping Yin ${ }^{1 \dagger}$, Xuan Yin ${ }^{1}$, Anna Bogachko ${ }^{1}$, Tingting Liang ${ }^{1}$, Lixing Lao ${ }^{2,3^{*}}$ and Shifen $\mathrm{Xu}^{1 *}$ (D)
}

\begin{abstract}
Background: The National Institutes of Health estimates the prevalence of insomnia in menopausal women at 40$50 \%$. Some studies have shown that acupuncture might be effective in treating primary insomnia and insomnia related to depression and stroke. Although there are some programs supporting insomnia during the menopausal transition, there are few randomized controlled trials (RCT) to provide evidence regarding their effectiveness. We design a RCT of suitable sample size to verify the effectiveness of acupuncture in patients with insomnia during the menopausal transition and to form an optimized acupuncture treatment protocol.

Method/Design: In this randomized, single-site, single-blind, placebo-controlled trial, 84 eligible patients will be recruited and randomly assigned to either the acupuncture group $(n=42)$ or the sham control group $(n=42)$ in a 1:1 ratio. Participants will receive a total of 18 treatment sessions for eight consecutive weeks. Treatments will be given three times per week in the first four weeks, twice a week for the next two weeks, and finally once weekly for the final two weeks. Treatment will utilize eight main acupoints (GV20, GV24, GV29, RN6, RN4, SP6, HT7, EX-HN22) and extra two acupoints based on syndrome differentiation. The primary outcome will be assessed using the Pittsburgh Sleep Quality Index (PSQI). The secondary outcomes will be measured by sleep parameters recorded in the Actigraphy (SE, TST, SA), Insomnia Severity Index (ISI), Self-Rating Anxiety Scale (SAS), Self-Rating Depression Scale (SDS), and Menopause Quality of Life (Men-QOL). The primary outcomes will be assessed at baseline, week 4, week 8, and the first and third month after the end of treatment.
\end{abstract}

Discussion: If the results confirm that acupuncture is effective and safe for the treatment on insomnia in menopausal women, this positive outcome could provide evidence for clinical application.

Trial registration: Chinese Clinical Trial Registry, ChiCTR1800018645. Registered on 10 January 2018.

Keywords: Menopause, Insomnia, Acupuncture, Clinical trial

\section{Background}

The menopausal transition (MT) is often accompanied by a myriad of symptoms, the most common being insomnia [1-3]. Insomnia is characterized by having difficulty falling asleep, frequent nocturnal waking with difficulty falling back asleep [4], and unexplained pain. A study conducted by the National Institutes of Health estimates the prevalence of insomnia in menopausal

\footnotetext{
*Correspondence: Ixlao1@hku.hk; xu_teacher2006@126.com

${ }^{2}$ School of Chinese Medicine, University of Hong Kong, Hong Kong, China

${ }^{1}$ Shanghai Municipal Hospital of Traditional Chinese Medicine, Shanghai

University of Traditional Chinese Medicine, Shanghai 200071, China

Full list of author information is available at the end of the article
}

women at $40-50 \%$ [5-8]. Women who experience poor sleep quality during the MT are more likely to have critical problems [9] such as depression, anxiety, memory and cognitive deprivation, cardiovascular disease, and reduced quality of life $[3,4]$. Still, there are few effective therapies available for treating sleep problems during the MT. Yoga, therapeutic massage, and exercise may be helpful for sleep disorders, but they have not yet been proven as an effective independent treatment [10]. Hormone therapy (HT) is currently the most effective treatment but is also associated with a number of risks $[11,12]$. HT can reduce hot flashes at night; some studies have reported that it can reduce sleep disorders [13]. 
However, increasing numbers of women are refusing HT due to the risks (myocardial infarction, stroke, venous thromboembolism, and breast cancer) outlined by trials published in the Women's Health Initiative [14]. Moreover, these trails reported that HT has a weaker effect on insomnia than previously thought [15] .

Acupuncture, an important part of traditional Chinese medicine (TCM), has been used for thousands of years in China to treat various diseases including sleep disorders [5]. Many studies have confirmed the efficacy of acupuncture for primary insomnia and related depressive disorders [16-18]. Based on results of the efficacy of acupuncture for depression-related insomnia [19] and insomnia following stroke [20], we believe that acupuncture might be an effective treatment for insomnia during the MT. However, effects of acupuncture on sleep disorders remain uncertain because of flawed research methodology, such as improper sample size, treatment times, and follow-up problems [21].

The main objective of this study is to determine whether acupuncture is effective as compared to a sham acupuncture control on insomnia in menopausal women. The findings of this trial will provide useful information in forming an optimal acupuncture treatment protocol.

\section{Methods/Design}

Hypotheses

Acupuncture treatment helps menopausal transition insomnia patients improve their sleep quality and other related symptoms.

\section{Objectives}

1. To compare the differences in improvement of insomnia, assessed by the Pittsburgh Sleep Quality Index (PSQI) and Insomnia Severity Index (ISI), between the intervention group and control group.

2. To compare the differences in improvement of mood, measured by the Self-Rating Anxiety Scale (SAS) and Self-Rating Depression Scale (SDS), between the intervention group and control group.

3. To compare the differences in improvement of Menopause Quality of Life (MenQOL), between the intervention group and control group.

\section{Design}

This is a single-site, single-blind, randomized, placebocontrolled clinical trial that will be carried out in the Shanghai Municipal Hospital of Traditional Chinese Medicine. Eligible patients will be randomly divided into the acupuncture group and the sham acupuncture group in a 1:1 allocation ratio. All participants will sign the informed consent before proceeding with the trial. The flow chart of the study process is as follows in Fig. 1. Timing of treatment assessments and data collection are as follows in Table 1.

\section{Recruitment}

Participants of the study will be recruited through the outpatient clinic, hospital-based Wechat advertising, and posters in Shanghai Municipal Hospital of Traditional Chinese Medicine in Shanghai, China. Those who are interested in participating in the study will be screened either via telephone or on-site. They will receive eight weeks of acupuncture treatment or sham acupuncture and a series of assessments on sleep disorders free of charge. During the study, participants will be required to wear a wrist Actigraphy to record their sleep quality. The participants who meet the inclusion criteria will be informed of the research details and asked to sign the informed consent before the treatment commence. The research assistant will obtain informed consent from participants.

\section{Inclusion criteria}

Participants with the following conditions will be included:

(1) Menopausal women aged 45 60 years;

(2) Participants who meet the diagnostic criteria of TCM in accordance with the above perimenopausal and insomnia [22];

(3) Participants in line with the international classification of sleep disorders (ICSD-3) for the diagnosis of insomnia;

(4) Participants whose PSQI score is > 5;

(5) Participants who voluntarily agree to join the study and to sign the written informed consent form for the randomized controlled trial (RCT).

\section{Exclusion criteria}

Participants with the following conditions will be excluded:

(1) Participants who have severe mental illness such as depression, anxiety, schizophrenia, etc.;

(2) Participants with serious heart, brain, kidney, or liver disease;

(3) Participants with secondary insomnia caused by systemic diseases such as pain, fever, cough, surgery, and external environment disturbances;

(4) Participants who have taken hormones for $\geq 6$ months;

(5) Participants who are pregnant or currently lactating. 


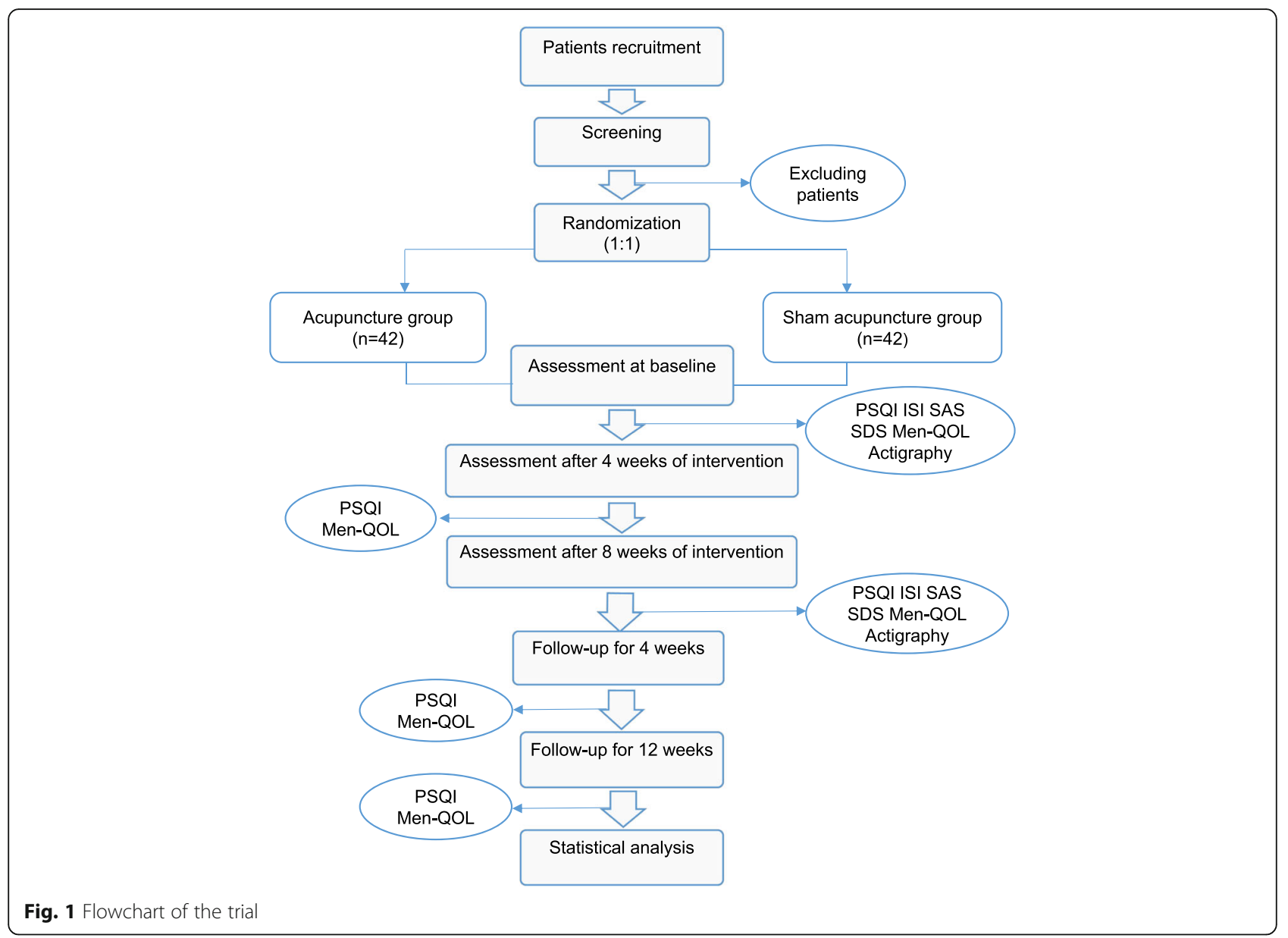

\section{Intervention}

Participants in each group will receive 18 real or sham treatments within an eight-week period. In each group, they will receive either acupuncture or sham acupuncture treatment three times per week for the first four weeks, twice per week for the next two weeks, and once per week for the last two weeks. Each treatment will last for $30 \mathrm{~min}$. Each patient will then be placed in a separate quiet space and lying supine. The acupuncturists will be registered practitioners with $>3$ years of experience in acupuncture practice. In order to increase the patients' compliance, they will accept the intervention and assessment by reservation and they will receive some financial subsidies (200 RMB) after the follow-up assessments.

In the treatment group, acupuncture needles will be standard stainless steel, sterile, and disposable $(0.25 \times 40 \mathrm{~mm}$ and $0.30 \times 40 \mathrm{~mm}$ in length; Jia Jian, China). The control group will use the Streitberger Placebo-needle at the same acupoints. The treatment methods of acupuncture and acupoints are shown in Table 2.

\section{The acupuncture group}

Participants in this group will receive acupuncture treatment during the first eight weeks. According to our in-depth research on insomnia and acupuncture experience, Baihui (GV20), Shenting (GV24), Yintang (GV29), Qihai (RN6), Guanyuan (RN4) and bilateral Anmian (EX-HN22), Sanyinjiao (SP6), and Shenmen (HT7) will be used as main acupoints [17]. Acupuncturists will be permitted to add acupuncture points based on TCM syndrome differentiation [23]. According to our own clinical practice, potentially relevant extraneous points include Mingmen (GV4) and Shenshu (BL23) for kidney yang deficiency and TaiXi (KI3) and Fuliu (KI7) for kidney yin deficiency. The needles will be inserted into the skin to the depth of $10-30 \mathrm{~mm}$ and manipulated manually (including lifting, thrusting, and rotating) until the patient reports needling sensations (Deqi sensation). The needles on GV20 and GV29 will be connected to a G6805-2 Multi-Purpose Health Device (Huayi Company), using continuous wave type, frequency at $2.5 \mathrm{HZ}$, and intensity of $45 \mathrm{~mA}$. Needles will be retained for 30 min before removal. 
Table 1 Timing of treatment assessments and data collection

\begin{tabular}{|c|c|c|c|c|c|c|}
\hline \multirow[b]{3}{*}{ Timepoint $^{a}$} & \multicolumn{6}{|c|}{ Study period } \\
\hline & \multirow{2}{*}{$\begin{array}{l}\text { Enrolment } \\
-1 \text { week }\end{array}$} & \multirow{2}{*}{$\begin{array}{l}\text { Baseline } \\
0 \text { weeks }\end{array}$} & \multicolumn{2}{|c|}{ Treatment phase } & \multicolumn{2}{|c|}{ Follow-up phase } \\
\hline & & & 4 weeks & 8 weeks & 4 weeks & 12 weeks \\
\hline \multicolumn{7}{|l|}{ Enrolment } \\
\hline Eligibility screen & $x$ & & & & & \\
\hline Informed consent & $x$ & & & & & \\
\hline Medical history & $x$ & & & & & \\
\hline Allocation & & $x$ & & & & \\
\hline \multicolumn{7}{|l|}{ Interventions } \\
\hline Acupuncture & & $x$ & $x$ & $x$ & & \\
\hline Sham acupuncture & & $x$ & $x$ & $x$ & & \\
\hline \multicolumn{7}{|l|}{ Assessments } \\
\hline \multicolumn{7}{|l|}{ Primary outcome } \\
\hline PSQI & & $x$ & $x$ & $x$ & $x$ & $x$ \\
\hline \multicolumn{7}{|l|}{ Secondary outcomes } \\
\hline$|S|$ & & $x$ & & $x$ & & \\
\hline Actigraphy & & $x$ & & $x$ & & \\
\hline SDS & & $x$ & & $x$ & & \\
\hline SAS & & $x$ & & $x$ & & \\
\hline Men-QoL & & $x$ & $x$ & $x$ & $x$ & $x$ \\
\hline \multicolumn{7}{|l|}{ Others } \\
\hline Estazolam dose & & $x$ & $x$ & $x$ & $x$ & $x$ \\
\hline Adverse events & & $x$ & $x$ & $x$ & $x$ & $x$ \\
\hline Patients' satisfaction & & & & & & $x$ \\
\hline Success of blinding & & & & & & $x$ \\
\hline
\end{tabular}

Table 2 Details of intervention

\begin{tabular}{lll}
\hline & Intervention group & Control group \\
\hline Main acupoints & GV20, GV24, GV29, RN6, RN4, SP6, HT7, EX-HN22 & GV20, GV24, GV29, RN6, RN4, SP6, HT7, EX-HN22 \\
Added acupoints & Kidney yang deficiency GV4, BL23 & Kidney yang deficiency GV4, BL23 \\
& Kidney yin deficiency KI3, KI7 & Kidney yin deficiency KI3, KI7 \\
Depth of insertion & GV20, GV24, GV29, RN6, RN4, EX-HN22 10 mm & No insertion \\
NT7, SP6, GV4, BL23, KI7, KI3 15 mm & \\
Needle type & Steel needle (Wuxi Jiajian Medical Co. Ltd., Wuxi, China) & Blunt-tip needle (Streitberger Placebo-needle) \\
Needle sensation & With de-qi sensation & Without de-qi sensation \\
Electric stimulation & Needle on GV20, GV29 connected to G6805-2 Multi-Purpose & Needle on GV20, GV29, connected to G6805-2 \\
& Health Device (Shanghai Medical Instruments High-Techno, & Multi-Purpose Health Device (Shanghai Medical \\
China) with electric pulse at a frequency of 2.5 Hz and an & Instruments High-Techno, China) without electric pulse \\
intensity of 45 mA & \\
& Three times per week for the first four weeks & Three times per week for the first four weeks \\
& Twice per week for the next two weeks & Twice per week for the next two weeks \\
& Once per week for the final two weeks & Once per week for the final two weeks \\
\hline
\end{tabular}




\section{The control group}

Participants in this group will receive sham acupuncture treatment. We will use a non-invasive placebo control, the Streitberger Placebo-needle. Acupoints are the same as with acupuncture group, without insertion. An electroacupuncture apparatus (G6805-2 Multi-Purpose Health Device) will be set beside the patients and connected to the GV20 and GV29, without electrical pulse. Needles will be also retained for $30 \mathrm{~min}$ before removal.

\section{Outcome measures}

We will assess the primary outcome at baseline, week 4 , week 8 , after treatment, and with follow-ups at week 4 and week 12 after the end of treatment. Secondary outcomes will be assessed at baseline and week 8 .

\section{Primary outcome}

\section{Pittsburgh Sleep Quality Index (PSQI)}

The PSQI is a self-rated questionnaire used to measure general sleep quality. It comprises 19 self-rated items and five other-rated items [20]. It assesses sleep based on seven domains in the past month: sleep duration; sleep disorders; sleep-onset latency; daytime dysfunction; sleep efficiency; use of medications to sleep; and overall sleep quality [24]. Each domain is rated 0-3; the accumulated scores of the seven domains constitute the total score of the PSQI $(0-21)$. A total score of $>5$ indicates a poor quality of sleep [25] .

\section{Secondary outcomes}

\section{Insomnia Severity Index (ISI)}

The ISI is designed to assess the nighttime and daytime symptoms of insomnia. It includes seven items rated on a scale of 0-4 points; the total score is in the range of 0-28 [26]. All self-reported questionnaires are conducted in Chinese. A higher score indicates a more severe insomnia. ISI classification: clinical insomnia (score 0-7), mild insomnia (score 8-14), moderate insomnia (score 15-21), and severe insomnia (score 22-28).

\section{Actigraphy assessments}

The wActiSleep-BT actigraph (Actigraph LLC, Pensacola, FL, USA), will be worn on the patients' wrist, which can record the sleep quality by noting sleep awakenings (SA), total sleep time (TST), sleep onset, sleep latency as well as the efficiency of sleep. The analysis of sleep condition and sleep quality will be performed by the software ActiLife6 (Version 6.8.1, Actigraph LLC) [17, 20].

\section{Self-Rating Anxiety Scale (SAS)}

We will use SAS to assess the degree of anxiety in patients. The questionnaire includes 20 items, as follows: 15 negative and five positive descriptions, the opposite of the score [27]. The total score of the 20 items is $>40$ points, indicating the state of anxiety. A higher score indicates more severe anxiety.

\section{Self-Rating Depression Scale (SDS)}

The SDS is a self-rated scale for evaluating the severity of depression [28]. The main statistical index consists of 20 questions. We first calculate the standard score by ranking the participant's responses to 20 questions. From this figure, the total score determined as is 1.25 times the standard score, making use of the integral part [17]. The demarcation point of depression is 50 points, the higher the score, the more significant the depression tendency.

\section{Menopause Quality of Life (MenQOL)}

MenQOL is a questionnaire used to assess the quality of life in menopause, including 29 items, which are divided into four domains: Vasomotor (Items 1-3); Psychosocial (Items 4-10); Physical (Items 11-26); and Sexual (Items 27-29). For analyses, we convert the item scores to a score in the range of $1-8(\mathrm{NO}, 0-6)$. Each domain mean is in the range of $1-8$. The overall questionnaire score is the mean of the domain means. A higher score indicates a worse quality of life [29].

\section{Estazolam dose}

Patients will be allowed to take Estazolam $(0.5-2.0 \mathrm{mg})$ when they have difficulty in falling asleep for $>3$ consecutive days during the trial. They will be asked to record the dose and the time of taking Estazolam on the case report form (CRF).

\section{Sample size}

The sample size calculation was based on the change of PSQI scores. Systematic review [21] shows that at least a 2.70-point difference in PSQI scores between acupuncture and sham acupuncture has clinical significance. We expected that acupuncture would outperform the sham acupuncture by 3 points; therefore, a sample size of 35 participants should be recruited in each group. Considering a dropout rate of $\sim 20 \%$, each group will take 42 cases. Therefore, a total of 84 participants should be recruited for this RCT.

$$
\mathrm{N}=2 *\left[\frac{\left(U_{\alpha}+U_{\beta}\right) \sigma}{\delta}\right]^{2} ; \quad(\alpha=0.05, \beta=0.2)
$$

\section{Randomization and allocation concealment}

We will use the block randomization method. We plan to use SPSS version 23.0 software to generate a random number table. The participants who meet the criteria will be randomly assigned to acupuncture group or control group with 1:1 ratio. The random allocation 
sequence will be generated in a block. The block size will be randomized to six.

The treatment allocation codes will be enclosed in sequentially numbered opaque envelopes by an independent researcher; the research assistant will pick the envelope and give it to the acupuncturist when the participants have finished all baseline assessments and immediately begin the first acupuncture treatment. The principal investigator (PI), co-investigators (Co-Is), and independent outcome assessors will be blinded to the treatment assignment. Only the acupuncturists will know to which groups the participants belong.

\section{Blinding}

Participants will be told that they will be randomly assigned to either acupuncture treatment or acupuncture-like simulation treatment and will be asked to wear an eye-patch when they receive treatment. Only the acupuncturists will know the group assignments. The participants and other researchers (including the data analysts, outcome assessors, and statisticians) will be blinded to group allocation. In order to ensure the successful implementation of the blinding method, all researchers will be trained before the trial begins.

\section{Blinding success assessment}

After the final treatment session, the success of blinding will be tested by asking the participants the following question "When you volunteered for the study, you were informed that you had an equal chance of receiving traditional acupuncture or acupuncture-like simulation treatment. Our study is finished now, which style acupuncture do you think you are received?" Three choices will be provided for participants: acupuncture treatment group; acupuncture-like simulation treatment group; and uncertain group. If participants choose "uncertain," we will ask the reasons why they have made that assumption.

\section{Safety and undesirable effects}

Patients will be advised in the event of any undesirable effect after the application of acupuncture that they should discontinue acupuncture and contact their doctors; the doctor will diagnose and treat the adverse reactions. They will also be asked to contact the researcher who will have completed an undesirable effect form as well as the undesirable effect record log. Adverse events (AE) such as fainting, allergies, and pain may occur in acupuncture clinical trials [30]. If the participant faints, the needle will be immediately withdrawn. The patient will be returned to a supine position and given warm water or sugar water. The patient will be allowed to rest until they have made a full recovery. In the case of pain and allergies, the needle should be taken out immediately and treated according to the specific circumstances.

Any AE (include any discomfort, symptoms, or diseases occurring during the trial) will be reported by the patients and doctors after acupuncture treatment. All details of AEs will be reported in the CRFs. At the end of the study, we will analyze the influence of all events.

\section{Data management}

Patient characteristics will be recorded in CRFs, which will be stored in the researcher's work office, and codes and initials will be used instead of the participants' information to protect the participants' privacy. Quality of CRF completion will be monitored by the specified researcher. All the original RCT data will be entered into the ResMan Research Manager of the Clinical Trial Management Public Platform. The relevant users will be trained and the system will be tested before it is officially launched to ensure that the system meets the trial requirements. Only relevant personnel will receive the account number and password once the system is officially launched. The clinical supervisor will monitor the work of the clinical trial center at least once a month.

\section{Statistical analysis}

All data will be analyzed by intention-to-treat (ITT), including data from any participants who have dropped out of the RCT during the trial. All data will be entered twice by two different researchers to ensure the accuracy. If the data are found to be uncertain, the data supervisor will notify the researcher to respond with a data question form. If necessary, the statistician will send a data question form to the researcher and the researcher's answer should be filled in the form. The question form is returned to the statistician by the inspector. The statistical analysis of data will be carried out by SPSS 23.0 software. Descriptive characteristics of baseline statistics will be reported as the mean \pm standard deviation or median.

To analyze the primary outcome, the change of PSQI scores between baseline and week 8 will be calculated; comparisons between acupuncture group and sham-acupuncture group will be made using the Student's $t$ test. The rank-sum test is used for ranked data, while the 2 test is adopted to analyze categorical data. For other secondary outcomes, ISI, SAS, SDS, and MenQoL and Actigraphy assessments (TST and SA) between the two groups will be compared with the Student's t test or the Wilcoxon rank-sum test. Analysis of secondary outcomes are considered exploratory; they were not part of the power calculations. All tests are two-tailed. $P$ values $\leq 0.05$ will be considered statistically significant. 


\section{Monitoring}

To improve the quality of this RCT, the whole process will be conducted under the supervision of a qualified clinical trial expert and be carried out by Shanghai Municipal Hospital of Traditional Chinese Medicine. The Clinical Research Center of Drugs of the Shanghai University of Traditional Chinese Medicine will provide data monitoring with access to any interim results and will make the final decision to terminate the trial if necessary. It also identify problems in the project, if any; the center makes the decisions to change the details of this protocol and announce the persons conducting the trial by written notice after approval by the application ethics committee. In addition, a qualified clinical trial expert will be invited to monitor this study and the PI will take full responsibility and will make the final decision.

\section{Discussion}

Acupuncture treatment has been practiced in China for more than two millennia [31] and in recent years has been widely used in Western countries [32]. Recent studies have found that acupuncture can be used to relieve vasomotor symptoms during the MT [23], but have not yet established the effects of acupuncture on insomnia during the MT. This is a valuable clinical RCT aimed at treating insomnia in menopausal women. We use acupuncture and sham acupuncture [33] to evaluate the efficacy of acupuncture on improving sleep quality. In addition, we use a strictly RCT, utilizing the control group method to reduce test bias and ensure the reliability of the RCT.

The improvement on previous trials offered in this RCT is the method of point selection. Individualized treatment upon syndrome differentiation is the basis of TCM theory [23]; the selection of points is a question for the world acupuncture researchers [34]. To give some evidence for the effects of treatment upon syndrome differentiation, we set eight points as main points and add two acupoints based on individual differences in the syndromes. Another advantage is the use of Actigraphy. This wrist-worn sleep monitor can provide an objective assessment for the treatment effect [35]. This is very useful for us to compare and evaluate the change of patients' sleep quality before and after treatment.

Another innovation in this trial is the design of the treatment frequency. In the first month, we will give full dose (three times per week) treatment. After the first month, we will decrease the frequency of the acupuncture treatment. This will be beneficial for us to observe the acupuncture therapeutic effect and to achieve the purpose of maintaining the minimum therapeutic dose in the long term.

However, this RCT still faces some challenges. First, the single-blinded method. For clinical trials of acupuncture, it is inevitable that acupuncturists know about the acupuncture treatment or sham acupuncture treatment. All participants will be asked to wear an eye-patch and will be arranged in a separate quiet space during the treatment. Second, the application of the sham acupuncture method. The acupuncturists will receive several sessions of strict training before the study begins to ensure the proper sham acupuncture techniques. Finally, the challenge of participant compliance. We will provide patient outreach over the phone to arrange the treatment time reasonably and to improve the attendance rate of the patients. The researchers will conduct follow-up interviews by phone to record patient outcomes.

To achieve our clinical goals, we will strive to standardize every step of the study, including acupoints selection, acupuncture operation, needle apparatus, and therapists' clinical experience. We expect that this trial will provide strong positive evidence for acupuncture treatment of sleep disorders in the perimenopausal period.

\section{Trial status}

The first investigators' meeting took place on 31 August 2018. The RCT is in preparation now and will launch in November 2018. Recruitment is expected to end in late 2020.

\section{Abbreviations}

CRF: Case report form; EX-HN: Extra acupoints on head; GV: Governor vessel; HT: Heart meridian of hand Shaoyin; HT: Hormone therapy; ISI: Insomnia Severity Index; ITT: Intention-to-treat; KI: Kidney meridian of foot Shaoyin; MenQOL: Menopause Quality of Life; MT: Menopausal transition; PSQI: Pittsburgh Sleep Quality Index; RN: Ren meridians; SAS: Self-Rating Anxiety Scale; SDS: Self-Rating Depression Scale; SP: Spleen meridian of foot Taiyin; TCM: Traditional Chinese medicine; TST: Total sleep time

\section{Acknowledgements \\ Shifen Xu provided general support as the head of the Acupuncture Department in Shanghai Municipal Hospital of Traditional Chinese Medicine and was responsible for the design of this RCT and for communicating important protocol modifications to relevant parties. We would like to thank Dr. Philippa Hazlewood from the International Education College, Shanghai University of Traditional Chinese Medicine, for her editorial support.}

\section{Funding}

The study is partly supported by grants from Shanghai Committee of Science and Technology, China (No. 18401905200). Shanghai Hospital Development Center, China (No. SHDC12016124), Shanghai Municipal Commission of Health and Family Planning, China (No. 201640026) and State Administration of Traditional Chinese Medicine, China (Grant No. JDZX2015024).

\section{Availability of data and materials}

The trial results will be published as peer-reviewed scientific papers and poster or oral presentations in conferences. All data and protocol will be available beginning three months and ending three years after publication of the results. The trial data will be available from the corresponding author upon reasonable request.

\section{Authors' contributions}

The trial was designed and developed by SFX and LXL. The manuscript was drafted by SSL. The protocol was carefully revised and edited by SSL and PY. $X Y, A B$, and $T T L$ contributed to the discussion. All authors read and approved the final manuscript. 


\section{Ethics approval and consent to participate}

This RCT was approved by the Ethics Committee of Shanghai Municipal Hospital of Traditional Chinese Medicine on 18 September 2018 (certificate number 2018SHL-KY-09). The purpose, procedures, and potential risks of the $\mathrm{RCT}$ will be explained clearly to the participants. All participants shall give their written informed consent to the research assistant before joining the $\mathrm{RCT}$

\section{Consent for publication}

Not applicable.

\section{Competing interests}

The authors declare that they have no competing interests.

\section{Publisher's Note}

Springer Nature remains neutral with regard to jurisdictional claims in published maps and institutional affiliations.

\section{Author details}

'Shanghai Municipal Hospital of Traditional Chinese Medicine, Shanghai University of Traditional Chinese Medicine, Shanghai 200071, China. ${ }^{2}$ School of Chinese Medicine, University of Hong Kong, Hong Kong, China. ${ }^{3}$ University of Maryland School of Medicine, Baltimore, MD 21201, USA.

\section{Received: 1 February 2019 Accepted: 19 April 2019}

Published online: 30 May 2019

\section{References}

1. Prairie BA, Wisniewski SR, Luther J, Hess R, Thurston RC, Wisner KL, et al. Symptoms of depressed mood, disturbed sleep, and sexual problems in midlife women: cross-sectional data from the Study of Women's Health Across the Nation. J Womens Health. 2015;24(2):119-26.

2. Chiu HY, Hsieh YJ, Tsai PS. Acupuncture to reduce sleep disturbances in perimenopausal and postmenopausal women: a systematic review and meta-analysis. Obstet Gynecol. 2016;127(3):507-15.

3. Yazdi Z, Sadeghniiat-Haghighi K, Ziaee A, Elmizadeh K, Ziaeeha M. Influence of sleep disturbances on quality of life of Iranian menopausal women. Psychiatry J. 2013;2013:907068.

4. Sun D, Shao H, Li C, Tao M. Sleep disturbance and correlates in menopausal women in Shanghai. J Psychosom Res. 2014;76(3):237-41.

5. Cong Fu NZ, Liu Z, Yuan LH, Xie C, Yang WJ, et al. Acupuncture improves peri-menopausal insomnia: a randomized controlled trial. Sleep. 2017;40:1-8.

6. Slopien R, Wichniak A, Pawlak M, Slopien A, Warenik-Szymankiewicz A, Sajdak S. Disturbances of sleep continuity in women during the menopausal transition. Psychiatr Pol. 2015;49(3):615-23.

7. Berecki-Gisolf J, Begum N, Dobson AJ. Symptoms reported by women in midlife: menopausal transition or aging? Menopause. 2009:16(5):1021-9.

8. Jin F, Tao M, Teng Y, Shao H, Li C, Mills E. Knowledge and attitude towards menopause and hormone replacement therapy in Chinese women. Gynecol Obstet Investig. 2015;79(1):40-5.

9. Philip S, Eichling JS. Menopause related sleep disorders. J Clin Sleep Med. 2005; 1:291-300.

10. Tal JZ, Suh SA, Dowdle CL, Nowakowski S. Treatment of insomnia, insomnia symptoms, and obstructive sleep apnea during and after menopause: therapeutic approaches. Curr Psychiatry Res. 2015;1:63-83.

11. Hachul H, Garcia TK, Maciel AL, Yagihara F, Tufik S, Bittencourt L. Acupuncture improves sleep in postmenopause in a randomized, doubleblind, placebo-controiied study. Climacteric. 2013:16:36-40.

12. Avis NE, Coeytaux RR, Levine $B$, Isom S, Morgan T. Trajectories of response to acupuncture for menopausal vasomotor symptoms. Menopause. 2017; 24(2):171-9.

13. Huang MI, Nir Y, Chen B, Schnyer R, Manber R. A randomized controlled pilot study of acupuncture for postmenopausal hot flashes: effect on nocturnal hot flashes and sleep quality. Fertil Steril. 2006;86(3):700-10.

14. Hays J, Ockene JK, Brunner RL, Kotchen JM, Manson JE, Patterson RE, et al. Effects of estrogen plus progestin on health-related quality of life. N Engl J Med. 2003:348:1839-45.

15. Kronenberg F, Fugh-Berman A. Complementary and alternative medicine for menopausal symptoms- a review of randomized, controlled trials. Ann Intern Med. 2002;137:805-14.
16. Yin X, X J, Dong B, Ma J, Chen Z, Yin P, et al. Efficacy and safety of electroacupuncture on treating depression related sleep disorders: study protocol of a randomized controlled trial. Evid Based Complement Alternat Med. 2016;2016:1069597.

17. Yin $X$, Gou M, Xu J, Dong B, Yin P, Masquelin F, et al. Efficacy and safety of acupuncture treatment on primary insomnia: a randomized controlled trial. Sleep Med. 2017;37:193-200.

18. Yeung WF, Chung KF, Tso KC, Zhang SP, Zhang ZJ, Ho LM. Electroacupuncture for residual insomnia associated with major depressive disorder- a randomized controlled trial. Sleep. 2011;34:807-15.

19. Dong B, Chen Z, Yin X, Li D, Ma J, Yin P, et al. The efficacy of acupuncture for treating depression-related insomnia compared with a control group: a systematic review and meta-analysis. Biomed Res Int. 2017;2017:9614810.

20. Cao Y, Yin X, Soto-Aguilar F, Liu Y, Yin P, Wu J, et al. Effect of acupuncture on insomnia following stroke: study protocol for a randomized controlled trial. Trials. 2016;17(1):546.

21. Cheuk DK, Yeung WF, Chung KF, Wong V. Acupuncture for insomnia. Cochrane Database Syst Rev. 2012;9:CD005472.

22. Sateia MJ. International classification of sleep disorders-third edition: highlights and modifications. Chest. 2014;146(5):1387-94.

23. Avis NE, Coeytaux RR, Isom S, Prevette K, Morgan T. Acupuncture in Menopause (AIM) study: a pragmatic, randomized controlled trial. Menopause. 2016;23(6):626-37.

24. Backhaus J, Junghanns K, Broocks A, Riemann D, Hohagen F. Test-retest reliability and validity of the Pittsburgh Sleep Quality Index in primary insomnia. J Psychosom Res. 2002;53:737-40.

25. Buysse DJ, Reynolds CF 3rd, Monk TH, Berman SR, Kupfer DJ. The Pittsburgh Sleep Quality Index-a new instrument for psychiatric practice and research. Psychiatry Res. 1988;28:193-213.

26. Bastien $\mathrm{CH}$, Vallières $\mathrm{A}$, Morin $\mathrm{CM}$. Validation of the Insomnia Severity Index as an outcome measure for insomnia research. Sleep Med. 2001:2:297-307.

27. Li RX, Ma M, Xiao XR, Xu Y, Chen XY, Li B. Perimenopausal syndrome and mood disorders in perimenopause: prevalence, severity, relationships, and risk factors. Medicine. 2016;95(32):e4466

28. Zung WW. A Self-Rating Depression Scale. Arch Gen Psychiatry. 1965;12:63-70.

29. Lemieux J, Brundage MD, Parulekar WR, Goss PE, Ingle JN, Pritchard KI, et al. Quality of Life From Canadian Cancer Trials Group MA.17R: A Randomized Trial of Extending Adjuvant Letrozole to 10 years. J Clin Oncol. 2018;36:563-71.

30. Li YQ, Lu YY, Zhang J, Guo Y. Analysis on the situation of adverse reaction to acupuncture and acupuncture risk. Chin Acupunct Moxibustion. 2011; 31(8):764-8.

31. Burke A, Upchurch DM, Dye C, Chyu L. Acupuncture use in the United States- findings from the National Health Interview Survey. J Altern Complement Med. 2006:12:639-48.

32. Yeung WF, Chung KF, Yung KP, Ho FY, Ho LM, Yu YM, et al. The use of conventional and complementary therapies for insomnia among Hong Kong Chinese: a telephone survey. Complement Ther Med. 2014;22(5):894-902.

33. Lao L, Bergman S, Hamilton GR, Langenberg P, Berman B. Evaluation of acupuncture for pain control after oral surgery: a placebo-controlled trial. Arch Otolaryngol Head Neck Surg. 1999:125(5):567-72.

34. Yeung A, Mischoulon D, Alpert J, Ma Y, Fava M. Acupuncture for Major Depressive Disorder: has its efficacy been disproved? J Clin Psychiatry. 2007; 68:1617-22

35. Short MA, Gradisar M, Lack LC, Wright H, Carskadon MA. The discrepancy between actigraphic and sleep diary measures of sleep in adolescents. Sleep Med. 2012;13(4):378-84.

Ready to submit your research? Choose BMC and benefit from

- fast, convenient online submission

- thorough peer review by experienced researchers in your field

- rapid publication on acceptance

- support for research data, including large and complex data types

- gold Open Access which fosters wider collaboration and increased citations

- maximum visibility for your research: over $100 \mathrm{M}$ website views per year

At $\mathrm{BMC}$, research is always in progress.

Learn more biomedcentral.com/submissions 